\title{
Semantic feature knowledge and picture naming in dementia of Alzheimer's type: A new approach
}

\author{
Peter Garrard $^{\mathrm{a}, *}$, Matthew A. Lambon Ralph ${ }^{\mathrm{b}}$, Karalyn Patterson ${ }^{\mathrm{c}}$, \\ Katherine H. Pratt ${ }^{\mathrm{c}}$, John R. Hodges ${ }^{\mathrm{c}}$ \\ a Institute of Cognitive Neuroscience, Alexandra House, 17 Queen Square, London WC1N 3AR, UK \\ b Department of Psychology, University of Manchester, Oxford Road, Manchester M13 9PL, UK \\ ${ }^{\mathrm{c}}$ Medical Research Council Cognition and Brain Sciences Unit, 15 Chaucer Road, Cambridge CB2 2EF, UK
}

Accepted 19 August 2004

Available online 7 October 2004

\begin{abstract}
This study addresses continuing controversies concerning the nature of semantic impairment in early dementia of the Alzheimer type (DAT), and the relationship between conceptual knowledge and picture naming. A series of analyses of fine-grained feature knowledge data show that: (1) distinctive features of concepts were more vulnerable than shared; (2) the amount of attribute knowledge about a concept was associated reliably, and in a graded fashion, with the ability to name a picture of that item; (3) sensory features were differentially important in naming; and (4) the degree of disruption to different types of attribute knowledge did not vary between items from living and nonliving domains. These findings are discussed in the context of contemporary cognitive and computational models of semantic memory organisation.
\end{abstract}

(C) 2004 Elsevier Inc. All rights reserved.

Keywords: Semantic memory; Semantic features; Naming; Alzheimer's disease

\section{Introduction}

Difficulties with expressive language are among the earliest and most prominent symptoms of Alzheimer's disease. Patients commonly complain of "forgetting words," particularly the names of things or people, and a profound anomia may be observed on bedside or formal testing. Despite intensive study of this phenomenon over the past decade, considerable controversy persists over whether it results from degradation of specific semantic information, or from a more general disruption of organisation impairing access to structurally intact representations.

Some studies have demonstrated normal performance by DAT patients on implicit tests of semantic

\footnotetext{
* Corresponding author. Fax: +44 2079168517.

E-mail address: p.garrard@ucl.ac.uk (P. Garrard).
}

knowledge [such as those involving semantic priming (Nebes, 1989)] others a dissociation between preserved recognition of semantic associates and impaired judgments of their semantic relatedness (Grober, Buschke, Kawas, \& Fuld, 1985; Nebes \& Brady, 1990), and these findings have been interpreted as supporting the access hypothesis. Other authors, however, have argued equally strongly for a degradation account on the basis of selective loss of fine-grained semantic information (Chertkow \& Bub, 1990; Hodges, Salmon, \& Butters, 1992b), the importance of lexical frequency in name retrieval (Hodges et al., 1992b), and item-by-item consistency between tests (Chertkow \& Bub, 1990; Huff et al., 1986; Lambon Ralph et al., 1997).

A striking demonstration of the relationship between object naming and the status of underlying semantic representations came from a study of the definitions 
generated by a group of DAT patients in response to the spoken names of concepts (Hodges, Patterson, Graham, \& Dawson, 1996). This study showed that the quantity and richness of information produced for items that had been named significantly exceeded that which was generated for unnamed items. Moreover, a discrepancy between the numbers of physical and associative attributes produced for named vs. unnamed items was considered to imply that knowledge of an item's physical features was particularly important to production of its name.

Although these findings are of central importance to the access vs. degradation debate, there were problems with some of the methods used in the study. The first relates to the techniques used to elicit semantic attribute knowledge: subjects were asked "How would you describe a $[\mathrm{X}]$ to someone who has no idea what one is ?" and allowed $1 \mathrm{~min}$ to provide their definitions. Although they were vigorously encouraged to produce as much information as possible, both the time limit and the form of the instruction may have inhibited participants from producing much more than a core definition of the concept. The response "A large African animal with a trunk" meets the specifications of the task by drawing on only a fraction of the semantic information that is usually encoded about elephants. Although it might be true simply that no more is known about the concept, it is also conceivable that the subject, while knowing much more about elephants, either considers such an abbreviated definition to be adequate to the requirements of the task, or is prevented by anomia from expressing any more detailed information in verbal form. It could also be argued that the features missing from the patients' definitions were inaccessible, rather than degraded.

Another problem concerned the methods used to score definitions: two strategies were adopted - the first was to sum the amount of information produced for each item, and the second to judge whether or not the definition conveyed the "core concept." Although results from both scoring systems behaved in a similar fashion in the study, it seems clear that they are measuring somewhat different abilities, as the complexity of such "core definitions" and the overall number of attributes may vary between items (consider the relative ease of generating information and encapsulating the core meaning in response to the words "horse" and "spanner"). Finally, the Hodges et al. study considered naming as an all-or-none phenomenon, whereas analysis of the types of error made by patients suggests that naming failures vary substantially in the quantity and/or quality of information that they convey. The relationship between name production and semantic knowledge would thus be considerably strengthened by the demonstration of a graded relationship between error types in naming and semantic knowledge generated.

In this study, we propose and evaluate a somewhat more systematic approach to the quantitative analysis of verbal definitions, motivated by a feature-based model of concept representation. Feature-based theories of semantic memory assume that concepts are composed of subordinate elements, or features (McRae, de Sa, \& Seidenberg, 1997; Rosch, 1973; Smith \& Medin, 1981).

It has been argued that featural representations can become partially disrupted, resulting in a graded deficit of knowledge about individual items, and such models can also account for the evolution in naming responses made over time by patients with semantic dementia (SD). This was clearly illustrated by the longitudinal study of patient J.L. (Hodges, Graham, \& Patterson, 1995a). A sample of J.L.'s naming responses over a period of 18 months is reproduced in Table 1, and reveals a pattern of progressively generic response-types. Thus, in the early stages he correctly named the prototypical birds, but failed to distinguish them from the more unusual category members. Later, the names of even the most familiar exemplars (chicken and duck) were replaced with the generic label "bird," and then with the even more non-specific "animal." Confusions between birds and other living creatures (e.g., "dog" for duck, or "horse" for eagle) began to emerge still later in the course of the disease, while more fundamental confusions (e.g., "vehicle" for peacock) remained exceptional even at very late stages.

According to feature-based models, category-level knowledge is supported by information that is common

Table 1

Naming responses to pictorial stimuli from the category birds, made by patient J.L. over an 18-month-period

\begin{tabular}{|c|c|c|c|c|}
\hline & September 91 & March 92 & September 92 & March 93 \\
\hline chicken & + & + & + & animal \\
\hline duck & + & + & bird & animal \\
\hline swan & + & bird & bird & $\underline{\operatorname{dog}}$ \\
\hline eagle & duck & bird & bird & horse \\
\hline ostrich & swan & bird & cat & $\underline{\text { animal }}$ \\
\hline peacock & duck & bird & cat & $\underline{\text { vehicle }}$ \\
\hline penguin & duck & bird & cat & part of animal \\
\hline rooster & chicken & chicken & $\overline{\text { bird }}$ & $\operatorname{dog}$ \\
\hline
\end{tabular}

Cross category errors are underlined, and "+" denotes a correct response (From Hodges et al., 1995a). 
to many members of the same category (shared features), while exemplar-level knowledge requires information that is unique to a single member (distinctive features), so the data shown in Table 1 would suggest that degradation of the semantic network was characterised, at least in the early stages, by a selective impairment of distinctive information. The network described by Rogers et al. (2004) illustrates this idea at the computational level of analysis by exploiting differences in feature distinctiveness to produce responses that are analogous to the behaviour of J.L. and other patients with SD. Progressively greater degrees of damage cause concept representations gradually to become dominated by their shared elements. In effect, the representations encoded by the damaged model tend towards an 'average' or 'prototype' of their categories, and when required to map between semantic and phonological representations, the network produces semantic errors ("dog" instead of "cat") or generic responses ("animal" instead of "cat"), similar to those made by patients with SD.

Adopting a somewhat more empirical approach, Garrard, Lambon Ralph, Hodges, and Patterson (2001b) collected a large corpus of semantic features from a group of normal controls, and showed that when similarities among concepts were computed simply on the basis of their lists of features, they grouped into clusters that broadly reproduced the superordinate categories to which they are normally assigned (animals, birds, vehicles, etc.). Moreover, the absence of any appreciable overlap between the representations of living and nonliving concepts suggested that large amounts of damage would need to be introduced before cross-domain confusions could occur - a pattern suggested, again, by J.L.

Although suggested by many authors (Garrard et al., 2001a, 2001b; Laatu, Portin, Revonsuo, Tuisku, \& Rinne, 1997; McRae et al., 1997; Moss, Tyler, Durrant-Peatfield, \& Bunn, 1998; Warrington \& Shallice, 1984), evidence that breakdown of the semantic system in the human brain is characterised by a preferential disruption to distinctive semantic features has so far remained indirect. Again, this stems from the limitations of free generation of definitions as a method for assessing semantic memory at the level of feature knowledge. Even though Hodges et al. (1996) were careful to encourage patients to produce as much information as possible, Warrington (1975) and Laatu et al. (1997) used forced-choice questions, and Laws, Humber, Ramsey, and McCarthy (1995) employed a sentence verification paradigm to probe for the presence of specific feature knowledge, in all cases the task was either open-ended, or difficult to score in a manner that compared the patients' responses to a normal standard, or both.

Two important methodological modifications mean that the present study is less vulnerable to such criticisms. First, the set of semantic features expected for each concept was determined empirically in advance of testing, using the semantic feature corpus of Garrard et al. (2001b). This allowed knowledge of shared vs. distinctive, as well as sensory vs. non-sensory information to be compared both within and between patients. Secondly, in order to compensate for the problems associated with anomia, a two-part test procedure was used: participants were asked first to generate as much information as they could about each concept, and then probed for information that was not spontaneously generated. Credit was given for non-verbal attempts to convey information about semantic attributes (e.g., miming, gesturing or pointing).

Furthermore, whereas many studies in this arena have focused on SD, the patients assessed here were a group with DAT, a condition in which the semantic impairment tends to be milder, and the confounding anomia less severe. This also allowed us to investigate in detail how the integrity of the semantic representations influenced the naming responses. To this end, naming errors were considered as reflecting graded quality and quantity of information, ranging from "don't know" or no response, to generation of a pertinent circumlocution.

The key predictions of the present study were, therefore, as follows: first, that the degradation of conceptual knowledge will be characterised by a disproportionate loss of distinctive over shared features; and secondly, that if impoverishment of semantic knowledge is responsible for the anomia seen in patients with DAT, then a graded relationship will be evident between degree of information revealed by the naming response, and quantity of semantic knowledge produced in the probed attribute task.

The richness of the data obtained using these novel methods will allow two other related issues to be addressed. The first concerns the adequacy of standard neuropsychological measures, such as naming and word-picture matching, for defining subtle semantic deficits, particularly in patients with very early DAT. It is hypothesised that a detailed test of semantic feature knowledge based on a corpus derived from normal, age-matched volunteers, will provide a more sensitive means of quantifying mild deficits. The second relates to the proposal that the differential importance of sensory and functional features to concepts from living and non-living domains underpins the phenomenon of category-specificity that is sometimes seen in patients with semantic memory impairment - an idea often referred to as "the sensory-functional theory" (SFT) (McCarthy \& Warrington, 1988; Warrington \& Shallice, 1984). Although the differential importance of these two basic knowledge types across semantic domains was not supported in the overall analysis of the features in the normal database (Garrard et al., 2001b), a more sensitive method for examining this hypothesis may be provided by a test of the detailed knowledge of semantic 
features underlying concepts in patients whose semantic memory is at various stages of breakdown.

\section{Patients and methods}

The study group was selected from a larger group of patients presenting to a Memory Disorder Clinic at Addenbrooke's Hospital between 1992 and 1996, and willing to be enrolled into a longitudinal study of semantic memory and related cognitive deficits in DAT. The diagnosis of probable DAT was made according to the criteria developed by the National Institute of Neurological and Communicative Disorders and Stroke (NINCDS) and the Alzheimer's Disease and Related Disorders Association (ADRDA), which consist of inclusion and exclusion criteria (McKhan et al., 1984). All patients presented with progressive cognitive impairment, predominantly affecting memory, with a history of between 1 and 4 years.

Ten patients were enrolled for the present study, representing a spectrum of cases with relatively early and more advanced disease who were willing to co-operate with the intensive testing required. Demographic and basic neuropsychological indices are displayed in Table 2. The mean age of the subjects was 72.8 ( $S D$ 6.9). Half the subjects were in the mildly impaired range (MMSE score between 20 and 25) and half were moderately impaired (MMSE score between 14 and 19). All patients have been followed for at least 12 months and have demonstrated the expected decline in cognitive function.

In addition to standard neuropsychological measures shown in Table 2, all patients were tested using: (i) a modified version of the semantic test battery described by Hodges and Patterson (1995b) [for details, see Garrard, Lambon Ralph, and Hodges (2002)], and (ii) a newly devised probed test of fine-grained feature-knowledge of all the items in the semantic test battery. Performance on the semantic battery was contrasted with that of a group of 31 age- and education-matched controls selected from the MRC-CBU subject panel. A second control group $(n=20)$ was used for the test of attribute knowledge.

\subsection{Semantic battery}

These tests all employ a consistent set of stimulus items from the corpus of line drawings by Snodgrass and Vanderwart (1980), and are designed to assess central representational knowledge via different modalities of input and different types of response output. A battery that has been used extensively in other studies (Hodges, Patterson, Oxbury, \& Funnell, 1992a; Hodges \& Patterson, 1995b; Hodges et al., 1996) was updated to contain 64 items chosen to represent four living categories (eight domestic land animals; eight foreign land animals; eight fruits; eight birds), and four nonliving categories (eight small manipulable household items; eight large, non-manipulable household items; eight vehicles; eight tools). Thus, in addition to being larger, the new set of items also differs in that the categories of musical instruments and water creatures in the original battery were omitted. The 64 items form two subsets, each containing 16 living and 16 nonliving items, matched in one for concept familiarity (Snodgrass \& Vanderwart, 1980) and in the other for age of acquisition (Morrison, Chappell, \& Ellis, 1997). The following tests were used for the present study:

1. Category fluency for each of six categories (animals and household items were treated as single categories), with 1 min allowed per category.

2. Naming of all 64 line drawings without cueing.

3. Word-picture matching in response to a spoken word for all 64 items. Subjects are presented with picture arrays consisting of 10 items from the same category (e.g., land animals) and asked to point to the item named by the examiner.

Table 2

Standard neuropsychological results for patients and controls

\begin{tabular}{|c|c|c|c|c|c|c|c|c|c|c|c|c|}
\hline & Max. & DT & $\mathrm{JH}$ & RT & $\mathrm{AN}$ & $\mathrm{RB}$ & $\mathrm{JF}$ & VA & PL & GC & AT & Control means $(S D)(n=31)$ \\
\hline Age & $\mathrm{n} / \mathrm{a}$ & 66 & 70 & 59 & 73 & 72 & 81 & 80 & 79 & 71 & 77 & $68.5(7.1)$ \\
\hline Sex & $\mathrm{n} / \mathrm{a}$ & $\mathrm{f}$ & $\mathrm{f}$ & $\mathrm{f}$ & $\mathrm{f}$ & $\mathrm{m}$ & $\mathrm{f}$ & $\mathrm{f}$ & $\mathrm{m}$ & $\mathrm{m}$ & $\mathrm{m}$ & $\mathrm{f} 18, \mathrm{~m} 13$ \\
\hline $\mathrm{MMSE}^{\mathrm{a}}$ & 30 & 14 & 16 & 16 & 19 & 19 & 23 & 24 & 24 & 25 & 25 & $28.8(0.9)$ \\
\hline Extended MMSE ${ }^{\mathrm{b}}$ & 100 & 40 & 31 & 35 & 61 & 51 & 65 & 64 & 77 & 81 & 73 & $92.7(3.0)$ \\
\hline Graded naming test & 30 & $\mathrm{nt}$ & $\mathrm{nt}$ & $\mathrm{nt}$ & 11 & 1 & 8 & 13 & 16 & $\mathrm{nt}$ & 16 & $20^{*}$ \\
\hline Digits forward & $\mathrm{n} / \mathrm{a}$ & 5 & 5 & 4 & 6 & 8 & 7 & 8 & 8 & 8 & 5 & $7^{*}$ \\
\hline $\mathrm{RMT}^{\mathrm{c}}$ (words) & 25 & 17 & 10 & 12 & 17 & 17 & 16 & 8 & 15 & 12 & 17 & $24.5(1.0)$ \\
\hline RMT (faces) & 25 & 25 & 12 & 9 & 14 & 17 & 15 & 13 & 22 & 14 & 16 & $24.4(0.6)$ \\
\hline Rey ${ }^{\mathrm{d}}$ figure copy & 36 & 2.5 & 7.5 & 27 & 29.5 & 27.5 & 24 & 13.5 & 29 & 35 & 31 & $34.2(1.6)$ \\
\hline Rey figure recall (\%) & 100 & 0 & 0 & 0 & 0 & 0 & 0 & 0 & 0 & 11 & 6 & $53.3(14.4)$ \\
\hline
\end{tabular}

n/a, not applicable; nt, not tested.

${ }^{a}$ Mini Mental State Examination (Folstein et al., 1975).

b A more comprehensive screening battery for cognitive dysfunction (Mathuranath et al., 2000).

${ }^{c}$ Recognition memory test (Warrington, 1984).

d Rey Osterreith complex figure test.

* Equivalent to 50th percentile for this age group based on published norms. 
4. Sorting pictures and words at 3 levels. In this test the patient is asked to sort cards, each card printed with either the name or a picture of one concept, into groups defined by their living or nonliving status (level 1), by semantic category-e.g., fruit, vehicle, etc. (level 2), or by the presence or absence of specific subordinate attributes ${ }^{1}$ (level 3 ).

\subsection{Probed test of semantic attribute knowledge}

In this test the subject was asked to produce as much information as possible about 62 of the 64 items in the semantic battery, which were presented in spoken word form. The test instruction was simply: "Tell me everything you know about a ...," together with encouragement to mention: (i) general ways of describing the item, (ii) the parts of the item, and (iii) things that the item can do or ways in which it can be used. Examples were worked through using two items not in the test battery (table and fish). A standardised set of semantic attributes for the items in the battery had already been assembled from the definitions provided by a group of 20 normal volunteers (roughly matched for age and educational level to the DAT patient group) using the procedure described in Garrard et al. (2001b). This attribute set was used to quantify the patients' performance as follows. For each item, the associated semantic attributes (which ranged in number from 14 (Barrel) to 30 (Dog)) were listed on a score sheet, together with the proportion of subjects who generated it in the featurelisting task (feature dominance). Patients were credited with knowledge of an attribute if it was generated during testing. To overcome the problems associated with assessing the more anomic subjects in this way, similar credit was given for non-verbal responses (e.g., gesturing, imitating or pointing), using a lenient criterion of success. No time limit was imposed, but attribute generation for each concept was terminated when the patient either indicated that he or she could think of no more to say, or began to repeat previously generated attributes. At this point, the patient was prompted to demonstrate knowledge of the remaining attributes on the score sheet, using probe questions which were specific (but never in the form of direct yes/no questions). For instance, if a patient had not mentioned spontaneously that a tiger has stripes, the question "what markings does a tiger have?" was asked. Likewise, in the absence of the spontaneously generated information that a kangaroo jumps or hops, a patient would be asked "how does a kangaroo move?"

\footnotetext{
${ }^{1}$ These were: for living categories: (a) foreign vs. native (b) larger vs. smaller than a man (c) meat eating vs. non-meat eating; and for nonliving categories (a) mainly metal vs. mainly non-metal (b) wooden vs. no wooden parts (c) small enough to be carried in a pocket vs. too large to be carried in a pocket.
}

The acquisition of each patient's test data took place in a series of sessions (typically four or five) over a period of up to two months, each session lasting between 2 and $3 \mathrm{~h}$. An identical procedure was carried out on 20 age-matched control subjects (though the testing time required was considerably shorter). Wherever possible, patients were re-tested after an interval of 12 months. This was possible for 8 of the 10 DAT patients. The patients' attribute knowledge for an item was calculated as the proportion of the total number of attributes available for that item, with each attribute weighted by its dominance value. In the data analyses that follow, attribute knowledge is therefore expressed as the proportion of the dominance-weighted sum of available attributes generated either spontaneously or overall.

In accordance with standard practice, data analysis was performed both with subjects and items as the random factor, and results are quoted as F1 and F2, respectively. In this study, because there was greater statistical power associated with items $(N=62)$ than with subjects $(N=10)$, effects are more likely to achieve statistical reliability in the by-items analysis.

\section{Results}

\subsection{Overall performance on tests of semantics}

Table 3 displays the performance of each of the patients, at the initial testing round, on tests from the standard semantic battery, with cases arranged in ascending order of MMSE score. The scores indicate that four of the patients (DT, JH, RT, and RB), all in the moderately affected subgroup, displayed a significant degree of anomia, and that all patients with the exception of $\mathrm{JH}$ performed better on the less demanding semantic tasks (word-picture matching and sorting).

\subsection{Feature content of normal and patient definitions}

\subsubsection{Reliability and sensitivity of the method}

Individual patients' performances on the probed test of attribute knowledge at the first and second (where applicable) testing rounds, together with the mean and 95\% confidence limits of the total attribute knowledge scores obtained by the control group, are displayed in Fig. 1. Each patient's score is sub-divided into features that were generated spontaneously and those offered in response to the probe questions. It is clear from the figure that not even the most mildly affected patients performed within the control range. Furthermore, no patient produced much more than $50 \%$ of their total attribute knowledge in the spontaneous condition.

A 2 (mild vs. moderate DAT) by 2 (round 1 vs. round 2) $\times 2$ (spontaneous vs. total response) analysis of variance, showed that: (i) the scores at round 1 significantly 
Table 3

Scores on tests form the semantic battery for patients and controls

\begin{tabular}{|c|c|c|c|c|c|c|c|c|c|c|c|c|}
\hline & Max. & DT & $\mathrm{JH}$ & RT & $\mathrm{AN}$ & $\mathrm{RB}$ & JF & VA & PL & GC & $\mathrm{AT}$ & Control means $(S D)(n=25)$ \\
\hline Letter fluency (/min) & $\mathrm{n} / \mathrm{a}$ & 3 & NT & 3 & 7 & 10 & 6 & 3 & 12 & 11 & 5 & $13.6(2.7)$ \\
\hline Category fluency (/min) & $\mathrm{n} / \mathrm{a}$ & 2.5 & $<1$ & 2 & 7 & 2 & 6 & 6 & 8 & 9 & 6 & $15.1(3.2)$ \\
\hline Naming (living) & 32 & 16 & 2 & 18 & 28 & 11 & 25 & 30 & 30 & 23 & 26 & $31.1(0.9)$ \\
\hline Naming (nonliving) & 32 & 21 & 5 & 20 & 32 & 18 & 31 & 29 & 31 & 29 & 32 & $31.2(1.2)$ \\
\hline Word-picture matching (living) & 32 & 28 & 12 & 25 & 32 & 25 & 30 & 30 & 31 & 25 & 29 & $31.8(0.4)$ \\
\hline Word-picture matching (nonliving) & 32 & 27 & 8 & 24 & 32 & 29 & 31 & 31 & 32 & 32 & 32 & $32(0.2)$ \\
\hline Sorting III (words) & 140 & 130 & NT & NT & NT & 111 & 129 & 128 & 128 & 124 & 130 & $133.8(3.9)$ \\
\hline Sorting III (pics) & 140 & 123 & NT & NT & 132 & 125 & 124 & 130 & 129 & 123 & 132 & $134.7(4.1)$ \\
\hline
\end{tabular}

nt, not tested; n/a, not applicable.

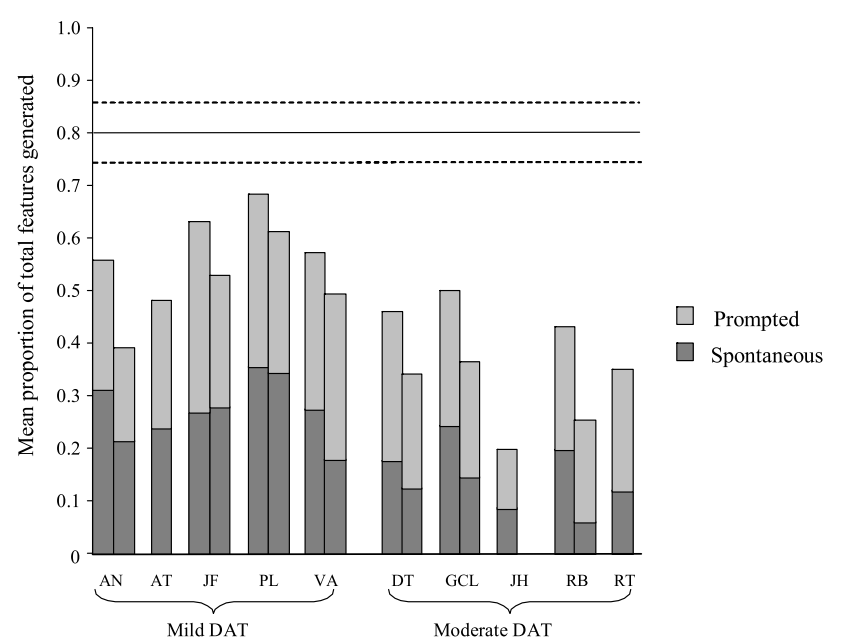

Fig. 1. Mean total feature knowledge per concept (proportion of the dominance-weighted sum of all the features associated with a concept) achieved by each of the subjects studied. Scores are divided into the components due to spontaneous and prompted generation of feature knowledge. Paired bars indicate scores at the first (left) and second (right) testing rounds. Reference lines show the mean and 95\% confidence limits of the total scores (i.e., after prompting) achieved by the control group.

exceeded those at round $2[F 1(1,6)=59.1, p<.001$; $F 2(1,61)=97.4, p<.001]$; (ii) the mildly affected patients scored consistently higher than the moderate group $[F 1(1,6)=10.9, p<.05 ; F 2(1,61)=675.1, p<$ $.001]$; and (iii) a significant amount of additional information was produced after prompting $[F 1(1,6)=85.9$, $p<.001 ; F 2(1,61)=823.1, p<.001]$. The marginal variation in longitudinal decline among the patient groups (group by round interaction) $[F 1(1,6)=2.7, p>.05$; $F 2(1,61)=33.9, p<.001]$, and the greater proportion of information produced after prompting by the moderate group (group by response type interaction) $[F 1(1,6)=2.1, \quad p>.05 ; \quad F 2(1,61)=23.1, \quad p<.001]$ reached significance only in the by items analysis.

\subsubsection{Effects of distinctiveness and intercorrelation}

Differences between the vulnerability of distinctive as compared with shared semantic features were sought by comparing the patterns of retrieval associated with fea- tures belonging to these two groups. Features were divided into relatively distinctive and relatively shared subsets (taken from the top quartile (0.06-0.125) and the bottom quartile (0.625-1.0), respectively, of the range of distinctiveness values). ${ }^{2}$ As before, each feature was weighted by its production frequency in the normal population (dominance). The mean proportions of features belonging to these two distinctiveness subsets generated at each testing round (either spontaneously or in response to a prompt question) are presented in Fig. 2, with mild and moderate DAT shown as separate subgroups.

These proportions were compared using a 2 (distinctive vs. shared) by 2 (testing round 1 vs. 2 ) $\times 2$ (mild DAT vs. moderate DAT) analysis of variance. As before, significantly more features were generated at the first testing round than at the second $[F 1(1,6)=179.4$, $p<.001 ; F 2(1,59)=115.9, p<.001]$. The by subjects analysis also showed that significantly more shared than distinctive information was generated by subjects from both groups at testing rounds 1 and $2,[F 1(1,6)=31.9$, $p<.01]$, though this difference failed to reach significance in the by items analysis $[F 2(1,59)=1.49$, $p>.05]$, as did all interactions involving distinctiveness. Subjects in the mild DAT group produced significantly less feature knowledge under both analyses $[F 1(1,6)=8.7, p<.05 ; F 2(1,59)=440.3, p<.001]$, and this difference may have been marginally more marked in the moderate subgroup (group by testing round interaction) $[F 1(1,6)<1 ; F 2(1,59)=15.7, p<.001]$.

The presence of a significant effect of distinctiveness in the by subjects, but not in the by items, analysis suggests that a subset of the items used in the test might have been contributing an atypical pattern of data. To explore this possibility further, the by items analysis was repeated using semantic domain as an additional two-level (living vs. manmade) between-items factor. This analysis confirmed a significant interaction between distinctiveness and domain $[F 2(1,58)=7.6, p<.01]$.

\footnotetext{
${ }^{2}$ According to the convention adopted in Garrard et al. (2001b), shared features have numerically higher distinctiveness values than distinctive features.
} 


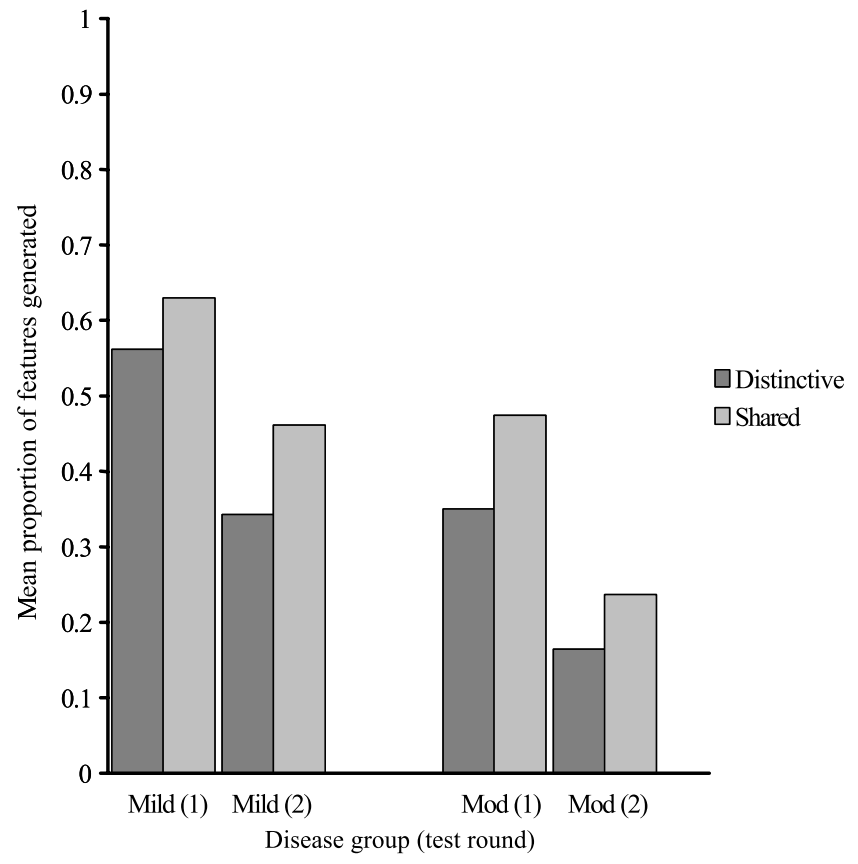

Fig. 2. Mean proportions of distinctive and shared features generated by patients from the two disease groups at the first and second testing rounds.

Inspection of Fig. 3, which displays the mean shared and distinctive feature knowledge scores from all patient testing sessions on the six basic level categories from which the stimuli were drawn, suggests that this interaction arose from the scores on items belonging to the category of household items. It has been noted previously
(Garrard et al., 2001b; McRae \& Cree, 2002; Rogers et al., 2004) that this category differs from the others, in part because of the relative poverty of shared structure among its features, a property which is also reflected in the patients' feature knowledge scores reported here.

This series of analyses suggests that damage to the semantic system in DAT affects distinctive rather than shared features to a disproportionate degree. One mechanism that has been postulated to explain this differential vulnerability employs the notion of feature intercorrelation. Intercorrelated features are those which are more likely to occur together than independently, and it has been suggested that this statistical fact might be reflected in a greater tendency for mutual activation between feature pairs. It is claimed that mutual activation provides a source of collateral support for such features, resulting in greater robustness in the face of damage (Devlin, Gonnerman, Andersen, \& Seidenberg, 1998; Gonnerman, Andersen, Devlin, Kempler, \& Seidenberg, 1997).

Correlation coefficients between every pair of features contributing to each of the six categories have already been calculated for the present data (Garrard et al. (2001a, 2001b)). Because the notion of intercorrelation implies a regular coactivation among feature pairs, stronger correlations are to be expected among features at the "shared" end of the spectrum of distinctiveness. This is confirmed in the present data by a positive correlation between the mean intercorrelation coefficient associated with each feature and its distinctiveness value $(R=.8, p<.001)$.

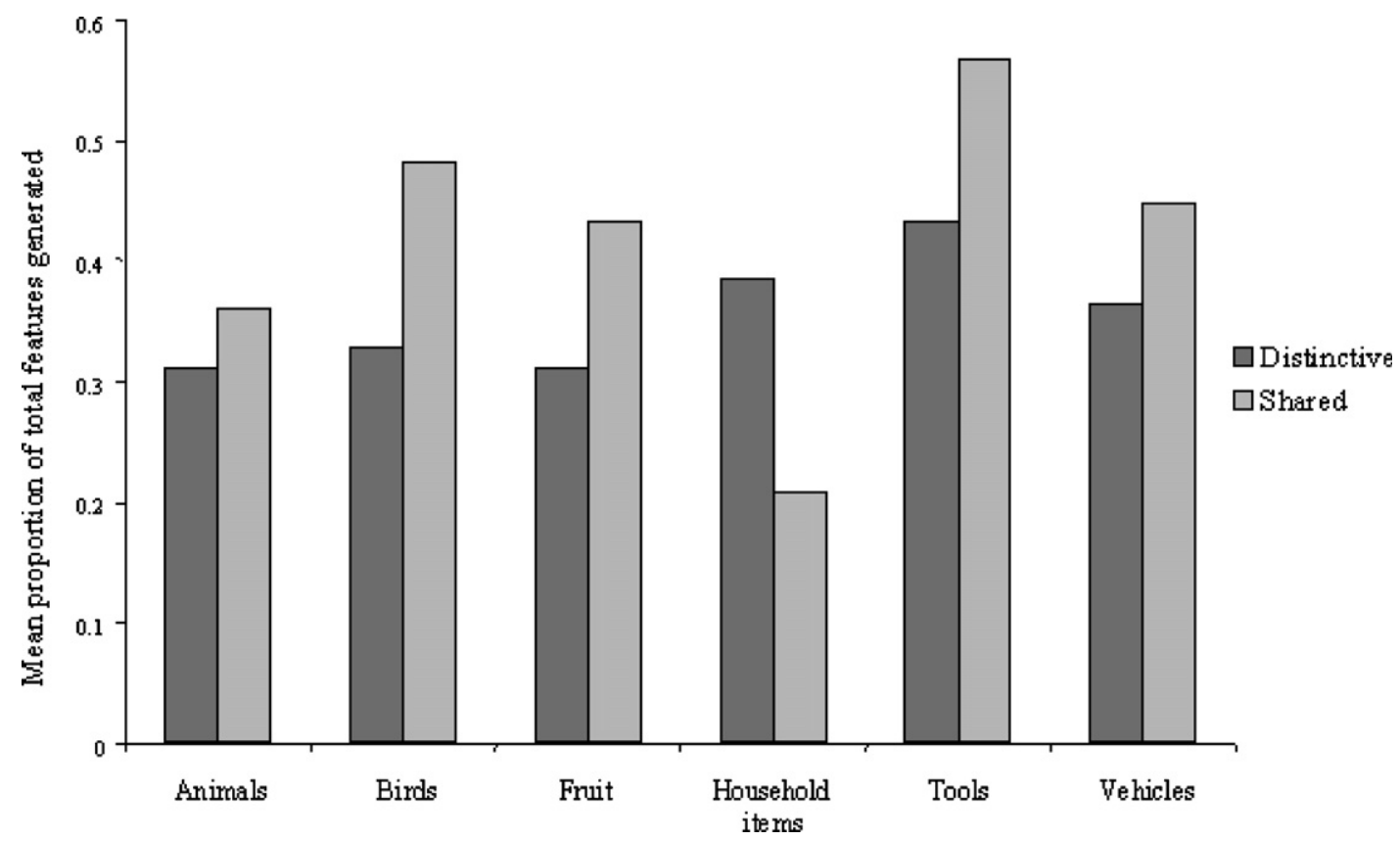

Fig. 3. Mean feature-knowledge scores from all patient testing sessions associated with items from the six basic-level categories from which the test stimuli were drawn. 
To examine the intercorrelation hypothesis further, the shared features were divided according to their tendency to form significant intercorrelations. For each feature we calculated the proportion of all features of concepts in the same semantic domain with which its correlation coefficient was statistically significant, as defined by the values of $R_{\text {critical }}$ for living and nonliving concepts (discussed in detail in Garrard et al., 2001b). This measure had a mean value of 0.08 ( $S D$ 0.04). Highly intercorrelated and relatively weakly intercorrelated subsets were defined by taking values falling in the top and bottom quartiles respectively of this distribution. The characteristics of these two subsets are contrasted in Table 4.

The mean scores (proportions of the total dominance-weighted sum of features generated) achieved by the two patient groups on these subsets of features are displayed in Fig. 4. Somewhat surprisingly, the scores relating to the minimally intercorrelated subset of features were consistently higher than those relating to the highly intercorrelated subset. A 2 (high vs. low inter- correlation) by 2 (testing round 1 vs. 2$) \times 2$ (mild DAT vs. Moderate DAT) analysis of variance confirmed that this difference was significant $[F 1(1,6)=10.9, p<.05$; $F 2(1,54)=5.6, p<.05]$, as (again) were the overall differences in feature-knowledge scores between testing rounds $[F 1(1,6)=20.4, p<.01 ; F 2(1,54)=97.8, p<$ $.001]$, and between mild and moderate patient groups $[F 1(1,6)=8.8, \quad p<.05 ; \quad F 2(1,54)=434.6, \quad p<.001]$. These two factors (testing round and patient group) interacted in the by items analysis $[F 1(1,6)=1.5$, $p>.05 ; F 2(1,54)=13.0, p<.01]$, and there was a small and similarly inconsistent three-way interaction $[F 1(1,6)<1 ; F 2(1,54)=6.8, p<.05]$.

\subsubsection{Comment}

This pattern of results suggests that feature intercorrelation is insufficient to explain the greater preservation of shared features in the present data. Although the minimally intercorrelated features were marginally but significantly less distinctive than those in the highly intercorrelated set (see Table 3), this difference is too

Table 4

Characteristics of two subsets of shared features from the test of semantic feature knowledge: a highly intercorrelated set consisted of those most often significantly correlated with other features belonging to concepts within the same domain (living or nonliving); a minimally correlated subset was associated with few such significant correlations

\begin{tabular}{lllr}
\hline Measure & Correlation group & & $F(1,349)$ \\
\cline { 2 - 4 } & High & Low & \\
\hline Proportion of significant intercorrelations & 0.13 & 0.04 & $403.9(p<.001)$ \\
Mean distinctiveness coefficient & 0.83 & 0.86 & $5.03(p<.05)$ \\
\hline
\end{tabular}

Correlations were calculated using Pearson's correlation coefficient.

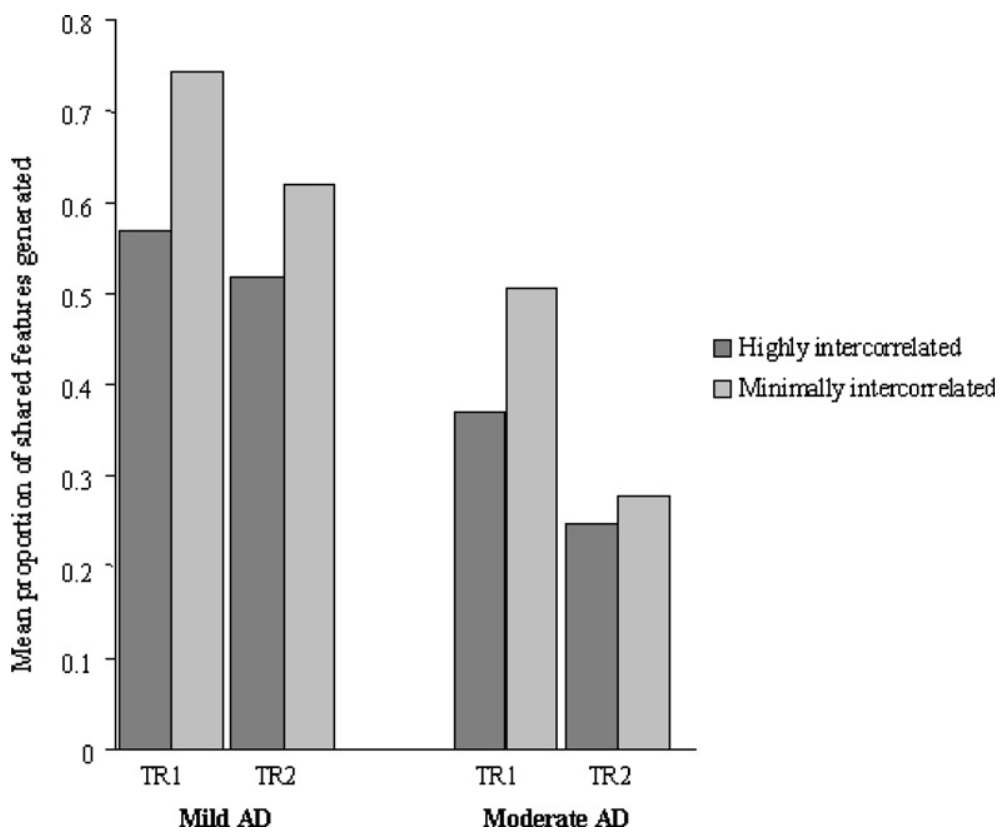

Fig. 4. Comparisons of the scores on the highly and minimally intercorrelated feature subsets achieved, at each testing round, by patient from each of the three disease groups. 


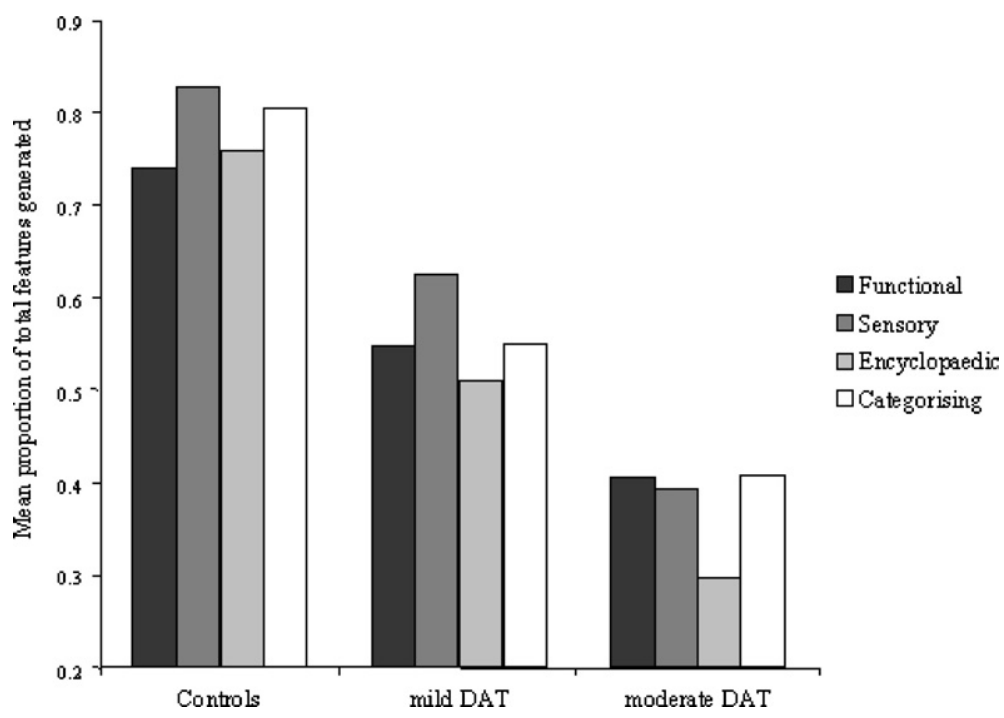

Fig. 5. Mean feature knowledge scores of each of the patient groups at the initial testing round, and the control group, with scores on the four feature subtypes shown separately.

small to be the sole explanation for the disparity. It could be argued that the finding is related to the systematic undersampling of shared features in the original feature production data, which almost certainly resulted in an underestimate of the true incidence of feature intercorrelations. On the other hand, there is no compelling reason to suppose a priori that such an undersampling would have affected intercorrelated features to a disproportionate extent.

\subsubsection{Interactions between feature type and semantic domain}

A third theoretically important aspect of the present data concerns the contributions of different feature types to the representations of items from different semantic domains. The analysis of data derived from feature-generation by cognitively normal subjects reported by Garrard et al. (2001b) showed that the proportional difference between the sensory and non-sensory features listed for concepts differed between living and nonliving concepts in a manner that was similar, though of smaller magnitude, to the findings of Farah and McClelland (1994). The findings of Garrard et al. (2001b) also raised the possibility that encyclopaedic features may have played a crucial role in the representations of concepts from living domains. The norms derived from that study provide an opportunity to examine in detail the relationship between the predictions of the sensory-functional theory (SFT) and the patterns of loss of different types of feature knowledge found in a series of patients at various stages of a neurodegenerative process.

Fig. 5 displays the mean proportions of the dominance-weighted totals of sensory, functional, encyclopedic and categorising features generated by subjects from the two patient groups at the initial testing round, ${ }^{3}$ and by the control group. These data were analysed using a 3 (controls vs. mild DAT vs. moderate DAT) $\times 4$ (sensory vs. functional vs. encyclopaedic vs. categorizing features) analysis of variance, which showed significant differences among the three subject groups $[F 1(2,27)=$ $109.2, \quad p<.001 ; \quad F 2(2,112)=304.9, \quad p<.001], \quad$ and among the four feature types $[F 1(3,81)=19.2$, $p<.001 ; F 2(3,168)=12.5, p<.001]$, as well as a significant interaction $[F 1(6,81)=4.8, p<.001 ; F 2(6,336)=$ $2.8, p<.05]$. Post hoc pairwise comparisons of the by subjects data, using Tukey's honestly significant difference test, confirmed that the scores of both patient groups were significantly lower than those of the controls on all types of knowledge. In addition, the moderate patients produced significantly less sensory and encyclopaedic feature knowledge than the those in the mild group, while functional and categorizing features were equal between groups.

Fig. 6 presents the mean sensory, functional and encyclopaedic feature knowledge scores achieved by subjects from the two patient groups and the control group, with values for living and nonliving items shown separately. To enhance any differences that may be present between the groups, these scores shown relate only to the distinctive features.

The data were analysed using a 3 (controls vs. mild DAT vs. moderate DAT) $\times 3$ (sensory vs. functional vs.

\footnotetext{
${ }^{3}$ Considering testing round as a separate factor would not only have limited further the number of complete cases in the analyses that follow, but would also have introduced an additional (and arguably redundant) level of complexity into the interpretation of the results. Regarding each test session as a separate observation, on the other hand, would have violated the assumption of independence.
} 


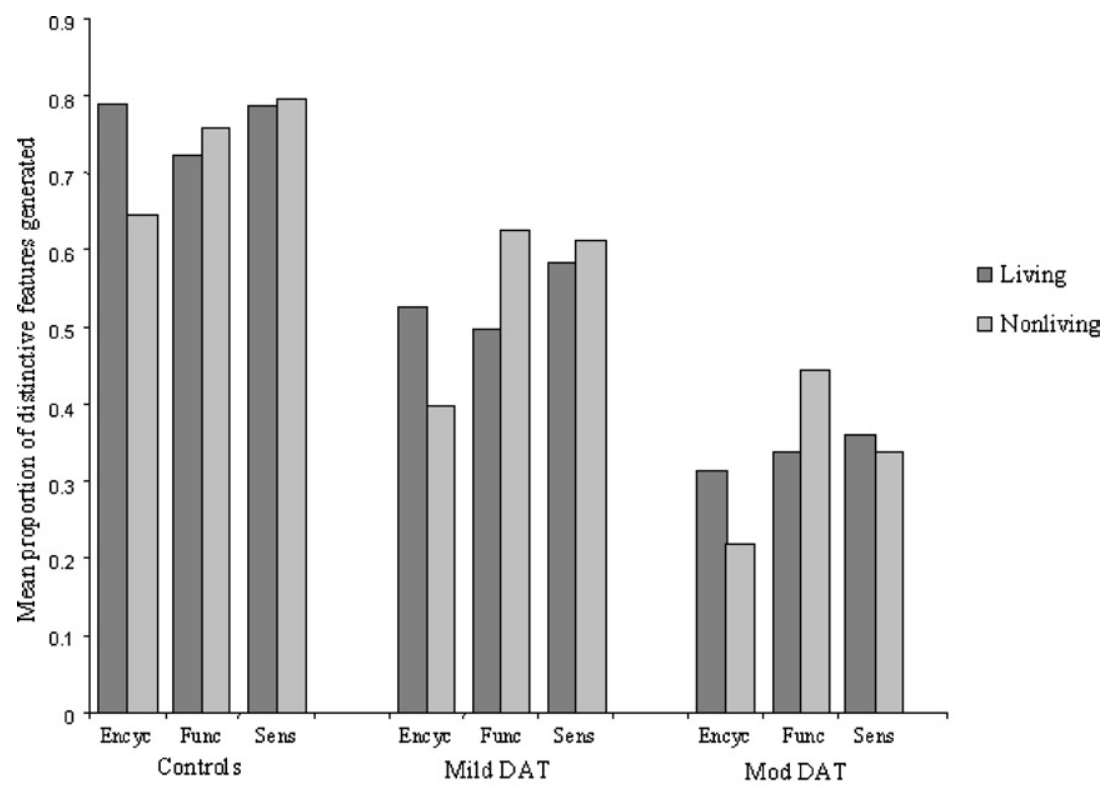

Fig. 6. Mean distinctive sensory, distinctive functional, and distinctive encyclopaedic feature knowledge scores associated with items from the living and nonliving domains.

encyclopaedic) $\times 2$ (living vs. nonliving) analysis of variance. This analysis confirmed main effects (noted above) of subject group $[F 1(2,27)=144.9, p<.001 ; F 2(2,92)=$ $319.8, p<.001]$ and feature type $[F 1(2,54)=53.8$, $p<.001 ; F 2(2,92)=6.6, p<.01]$. There was no consistent overall difference between the living and nonliving item subsets $[F 1(1,27)=1.2, p>.05 ; F 2(1,46)<1]$, but there were significant interactions between semantic domain and subject group $[F 1(2,27)=3.3, \quad p=.05$; $F 2(2,92)=3.8, p<.05]$ and (critically for this analysis) between semantic domain and feature type $[F 1(2,54)=107.7, p<.001 ; F 2(2,92)=5.4, p<.01]$. As before, the difference between the scores on the three feature types varied across groups, resulting in a significant interaction between these two variables $[F 1(4,54)=8.6$, $p<.001 ; F 2(4,184)=2.9, p<.05]$. A three-way interaction is suggested by: (i) the difference between patients and controls in relative functional knowledge scores for living and nonliving items, and (ii) the reversal-more marked in patients than controls - of the living-nonliving difference between encyclopaedic and other feature types. This three-way interaction was indeed significant $[F 1(4,54)=4.5, p<.01 ; F 2(4,184)=2.9, p<.05]$.

The finding of a significant interaction between feature type and semantic domain implies that the proportions of different types of feature knowledge varied between living and nonliving concepts. To explore this interaction further, the scores were collapsed across the two disease groups, and the sensory, functional, and encyclopaedic feature knowledge scores relating to items from the living and nonliving domains compared. The only significant difference between living and nonliving domains was that associated with the functional features $[F(1,54)=11.3, p<.01]$, among which higher knowledge scores were associated with concepts in the nonliving domain. Combining the functional and encyclopaedic feature sets, however, resulted in the relative proportions of sensory and non-sensory features becoming indistinguishable $[F(1,54)>1]$. If there were systematic differences in the cognitive structure of concepts associated with living and nonliving domains as predicted by the SFT, a significant interaction between domain and knowledge type might have been expected from the study of semantic systems under degraded conditions. The feature knowledge scores of these groups of patients, therefore, provide no convincing evidence in favour of the SFT.

\subsubsection{Comment}

The above analyses have suggested that patients with DAT differed significantly from normal controls not only in respect of the extent of their feature knowledge, but also in the relative proportions of surviving features of different types. The analyses performed so far have shown significant differences between control and disease groups on knowledge of all feature types, but the post hoc comparisons (see p. 20 above) revealed differences between the mild and moderate DAT groups only for sensory and encyclopaedic feature types. The inconsistency with respect to feature knowledge preservation between disease stages might be predicted on anatomical grounds: temporal neocortical regions may be particularly important in the representation of sensory information, and it has been suggested that, in the majority of patients with DAT, the pathological process spreads from medial to lateral temporal regions before involving 


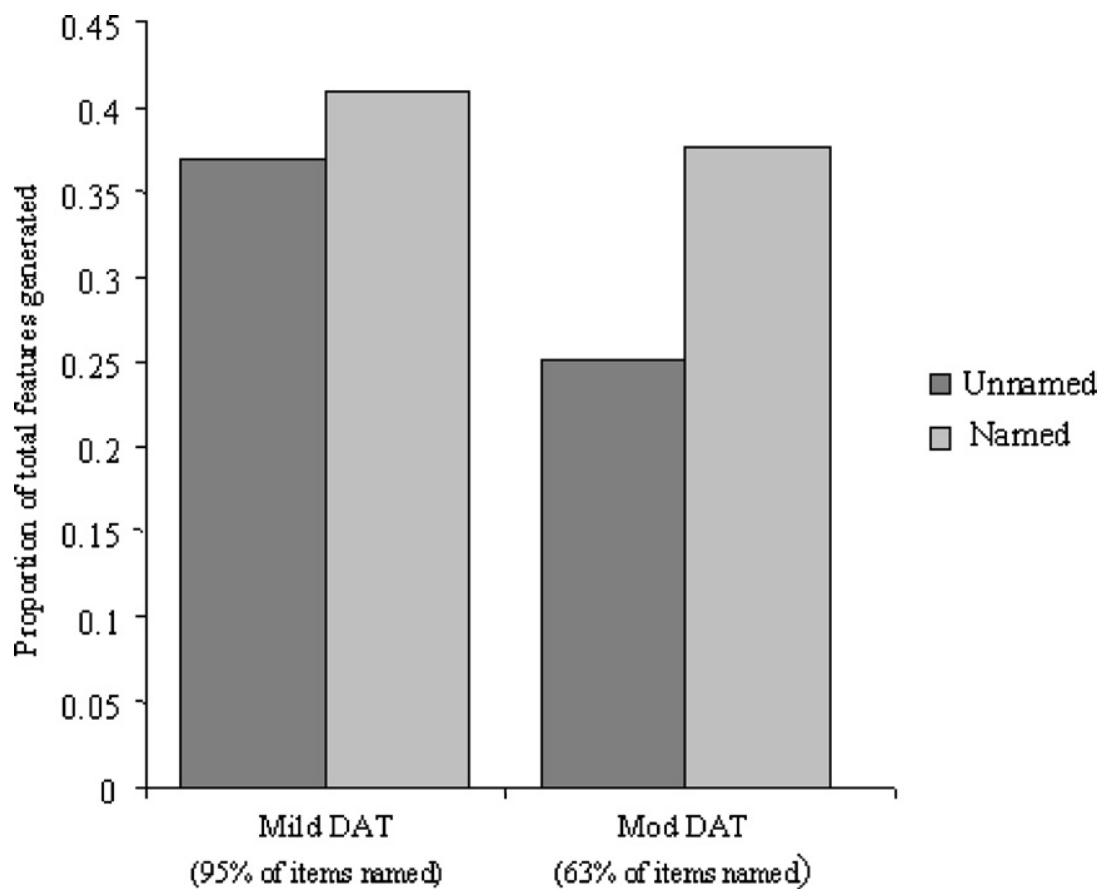

Fig. 7. Mean proportions of semantic features generated in response to named and unnamed items from the semantic battery by subjects with mild and moderate DAT at the first and second testing rounds.

posterior association areas (Braak \& Braak, 1991). This hypothesis will be considered further by comparing the proportions of features of each type produced for items that were correctly named to those produced for unnamed items.

\subsection{Relationship between naming and semantic feature knowledge}

To address the question of whether the naming deficit characteristic of DAT is due to degradation of specific semantic information, or to impaired access to relatively intact representations, the relationship between success at naming and amount of attribute knowledge generated was examined. Overall feature knowledge performance on the named and unnamed items is shown graphically in Fig. 7 which displays the mean feature knowledge scores achieved by the mild and moderate subject groups. Scores are collapsed across items, and divided according to whether the corresponding picture was correctly named. Because three patients had dropped out of the study after the first testing round, data from testing round 2 was not included in these analyses. ${ }^{4}$

A two (mild vs. moderate) by two (named vs. unnamed) ANOVA was used to analyse these results. In these analyses, although data from all cases are included, the $F$-ratio and hence the degrees of freedom

\footnotetext{
${ }^{4}$ Of the patients who were not retested, one (AT) died, and the other two withheld consent.
}

of the error term, are based on the number of complete cases (i.e., cases associated with data at all levels of all factors). This method of dealing with missing values in multivariate data is discussed in Everitt and Dunn (1991, p. 40).

In the by subjects analysis ( 10 complete cases) the difference between the two groups approached significance $[F 1(1,8)=3.4, p=.10]$, but there was no overall difference between scores associated with the named and unnamed items, nor any interaction $[F 1(1,8)<1]$ for both analyses. The by items analysis (27 complete cases), however, showed that the mild group produced reliably more attribute information than the moderate group $[F 2(1,25)=11.5, p<.01]$, and that significantly more information was produced overall about the named than the unnamed items $[F 2(1,25)=23.1, p<.001]$. The interaction between naming status and disease stage was also significant $[F 2(1,25)=5.54, p<.05]$.

\subsubsection{Comment}

These results replicate those obtained by Hodges et al. (1996): while confirming the variability in degree of anomia exhibited by individuals with $\mathrm{AD}$, they suggest that more demanding tests of semantic memory may be performed at below normal levels by all patients, and that disease stage is reliably associated with test performance. The data also suggest that there is a principled relationship between the status of semantic knowledge and the anomia that characterises many of the more impaired patients' neuropsychological profiles. 
As previously noted, this analysis neglects a number of potentially important factors. First, the possibility that distinctive information may be particularly crucial to name production was explored by considering subsets of attributes in the top and bottom quartiles of the range of distinctiveness values. Secondly, because picture naming is visually mediated, the attributes most related to naming success might be expected to be those that can be ascertained through the visual modality (i.e., mainly sensory attributes). Features were therefore further subdivided into sensory and functional subsets, with the small number of encyclopaedic features (which on this account would be expected to have little or not impact on the process of name retrieval) excluded from further analysis.

Finally, the naming errors characteristically made by DAT patients range from simple "don't know" responses to semantically rich and correct circumlocutions, such as "that Australian animal that jumps and carries its young in a pouch." Incorrect naming responses therefore seem to be more accurately characterised in terms of grades of information, rather than a simple dichotomy, and it could be hypothesised that failures of different kinds, or at different stages of the naming process, may underlie different types of erroneous naming response. According to this account, only items associated with certain types of naming error would differ from those named correctly in terms of the amount of semantic information retrieved in response to the concept name. If, on the other hand, semantic memory is playing a role in all types of naming error made by
AD patients, then a graded effect of semantic attribute knowledge would be predicted. The incorrect naming responses that convey most information should be associated with more attribute knowledge in the definitions task, and the most impoverished with significantly poorer definitions. The best definitions should correspond to correctly named items. Naming errors were therefore divided into three types, which were hypothesised to reflect increasing degrees of knowledge about the items to be named: (1) "don't know," semantically unrelated or incorrect circumlocutory responses; (2) generic category words (e.g., "animal," "fruit," etc.); and (3) close category-coordinates (e.g., "lion" for tiger, or "emu" for ostrich), or accurate circumlocutions.

\subsubsection{Effects of feature distinctiveness, feature type, and quality of responselerror}

The proportions of sensory and non-sensory attributes generated for items associated with each of the four types of naming response are shown in Fig. 8, with values for distinctive (8a) and shared (8b) attributes shown separately. A three-way, repeated measures ANOVA was used to examine the mean proportions of semantic feature knowledge associated with feature distinctiveness, feature type and naming response type (error types 1,2 , and 3 , and correct). In the by subjects analysis ( 5 complete cases available), there was significantly more sensory compared with functional knowledge $[F 1(1,4)=22.08, p<.01]$, but no other significant main effects. A small interaction emerged between knowledge type and distinctiveness $[F 1(1,4)=8.64$,

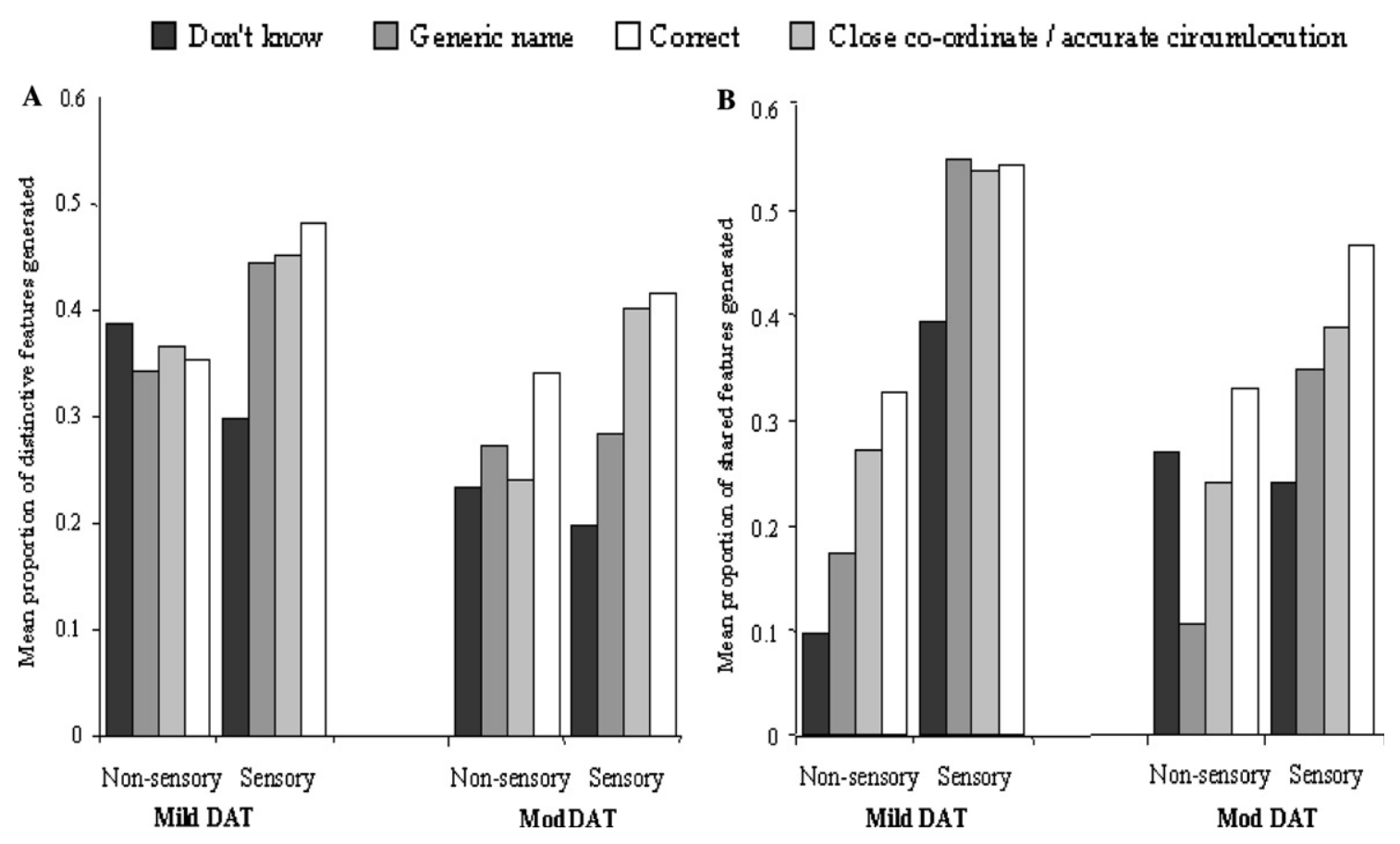

Fig. 8. Mean proportions of distinctive (A) and shared (B) sensory and non-sensory features associated with concepts identified at four levels by subjects in the three groups. 
$p<.05]$ reflecting the greater number of shared sensory features and of distinctive functional features. None of the interactions involving level of naming response reached significance. In the by items analysis (seven complete cases), there were significant main effects of feature type $[F 2(1,6)=9.09, p<.05]$, reflecting the larger number of attributes classified as sensory knowledge, and of naming response type $[F 2(3,18)=4.72, p<.05]$, as well as a significant interaction between these two factors $[F 2(3,18)=3.74, p<.05]$, but no effect of distinctiveness, nor any interactions involving this term.

To explore further the interaction between feature type and naming response type, separate 2-way (distinctiveness by naming response type) by-items ANOVA's were performed on the sensory and functional attribute subsets (seven complete cases in each). Among the sensory attributes, the slight overall excess of shared feature knowledge was not statistically significant $[F(1,6)<1]$, though the difference among the four naming response types was highly significant $[F(3,18)=5.35, p<.01]$. There was no interaction between these two factors. By contrast, among the functional attributes, neither of the main effects $([F(1,6)<1]$ for distinctiveness; $[F(3,18)=2.42, p>.05]$ for naming response type) nor the interaction $[F(3,18)<1]$ was significant.

\subsubsection{Comment}

These results provide some evidence that the more fine-grained semantic knowledge (as determined by probed definitions to concept name) that is retained about an item, the more detailed and appropriate the subjects' responses (even if erroneous) will be on tests of picture naming, and that this relationship applies particularly to knowledge of the item's sensory attributes. Although these conclusions are not statistically supported in the by-subjects analysis, the small size, as well as the considerable variability in the naming performance of the study population, makes the latter form of analysis rather insensitive. There was no clear evidence, generalizing across either subjects or items, that distinctive information is significantly more associated with correct naming responses than shared information, again possibly the result of insufficient statistical power.

\section{Discussion}

The data reported in this study of semantic memory in DAT were obtained using a novel test of semantic feature knowledge, which has a number of advantages over previously reported methods of assessing the integrity of conceptual knowledge at the fine-grained level. Most important, the test was based on a standardised database of semantic features derived from a baseline task performed by cognitively normal age-matched volunteers (Garrard et al., 2001b). This not only provided an objective criterion for quantifying feature knowledge, but also allowed the important variables of production frequency (dominance), distinctiveness, and intercorrelation to be incorporated into analyses of feature knowledge status in patients. Moreover, the method of probing for knowledge of features that were not spontaneously declared, at least partially overcomes the problem of word retrieval impairment that always hampers the assessment of fine-grained knowledge in tasks requiring speech output.

The detailed assessments performed have provided a large volume of empirical data from patients with impaired semantic memory, allowing a range of theoretically important factors to be systematically analysed. The principal findings from these analyses have been: (i) that the method of quantifying residual semantic knowledge by means of structured definitions with specifically guided probe questions, designed to elicit a defined corpus of feature knowledge, is a viable and sensitive means of assessing semantic memory in mild to moderate DAT; (ii) that in the course of progressive degradation of conceptual knowledge in these patients the distinctive features of individual items are marginally more vulnerable than the shared, and that this difference in feature vulnerability does not require additional assumptions about feature intercorrelation to be accommodated within a distributed model of semantic memory; (iii) that the level of attribute knowledge provided to concept name achieved for each item in the battery was reliably associated with the ability to name a picture of that item; and (iv) that while attribute types (sensory or functional) were differentially important in naming, the main prediction of the sensory-functional theory (namely, that the degree of disruption to different types of attribute knowledge should vary between concepts from the living and nonliving domains) was not upheld.

The first of these conclusions is justified by the observation that even the most mildly affected patients scored well below the lower limit of the control range, and that all subjects showed the expected decline in performance when reassessed at intervals of around one year. Moreover, the fact that patients' scores on knowledge of concept features were considerably enhanced by specific probe questions, further suggests that the extent of semantic knowledge is significantly underestimated by studies that rely purely on the content of freely generated concept definitions. It should, nonetheless, be noted that testing was long and arduous, and the influence on overall performance of non-specific factors such as fluctuating attention and cognitive slowing was not ruled out by the comparison with a cognitively normal control population.

The second finding (the differential vulnerability of distinctive features) may be attributable, in a connectionist framework, to the relatively greater influence of 
more commonly recurring elements on learned representations. It has been argued that this phenomenon can account for the characteristic pattern of semantic breakdown in which picture naming responses become progressively dominated by generic or highly prototypical terms, such as "animal" or "dog" (Hodges et al., 1995a; Warrington, 1975), but no previous studies have demonstrated it directly in patients with degraded semantics. Admittedly, the differences attributable to distinctiveness were small and only apparent in a byitems approach to the data, and it is certainly plausible that our assumption that the semantic system is organized as a distributed feature network is too simplistic. Nonetheless, relative undersampling of shared features in the standard feature set and/or the atypical feature structure associated with some categories almost certainly contributed to this inconsistency (see comments on pp. 16 and 17).

A similar absence of direct evidence bedevils the hypothesis that feature intercorrelation, rather than feature sharing per se, is critical to the vulnerability of distinctive attributes (Devlin et al., 1998; McRae et al., 1997); our shared feature analysis comparing those that were correlated, and those that were uncorrelated provided no evidence in favour of this view.

The final two sets of results replicated those of Hodges et al. (1996), who also found a relationship between naming and semantic knowledge as measured by the quantity and quality of spontaneously generated definitions. The methods used in the present study have, however, gone some way towards eliminating the component of attentional and executive dysfunction that could have accounted for at least part of patients' poor performance. Whereas in that study it was argued that many patients with mild DAT perform normally on demanding dual-task tests of frontal "executive" ability (Greene, Hodges, \& Baddeley, 1995), it is now clear that attentional deficits can be detected in a majority of DAT patients if a sufficiently wide range of neuropsychological instruments is used (Perry \& Hodges, 1999). The method of probing for specific attributes helps to control for attentional factors as well as for the confounding factor of anomia; and the demonstration that all patients provided significantly more semantic feature information when probed argues strongly for the involvement of one or both of these confounds in the spontaneous condition.

The idea that semantic memory degradation underpins anomia in DAT is given further support by the finding that the quality of the naming response is associated in a graded fashion with underlying attribute knowledge. That is to say, the more impoverished a patient's definition in terms of semantic attributes, the less appropriate or content-bearing will be his/her naming response to the corresponding picture. Thus, while it is possible to argue that, even with our technique of prob- ing for knowledge, naming deficits could still reflect either degraded knowledge or impairment of access, the data relating naming error type with amount and type of attribute knowledge produced make this argument much more difficult to sustain.

It is perhaps surprising that the distinctiveness of an attribute did not appear to have a direct effect on the strength of this relationship between knowledge and naming. The distinctive attributes are, after all, those which differentiate a concept from its category co-ordinates - which is essentially the role of the verbal label itself. One possible explanation for this may simply lie in the flexibility with which a concept name is assigned to a level in the hierarchy of superordinate terms (the term "cat," for example, designates a broader class of creatures when one is in a zoo), or in the variations in this hierarchical structure between individuals with different types of premorbid expertise.

An analysis of naming ability based on the success or failure of the patient to produce the "core concept" described by Hodges et al. (1996), might appear at first sight to have described an association between naming and knowledge of distinctive features. Yet scrutiny of some of the definitions which were judged to have conveyed the core concept, (such as Rhinoceros: "A very large animal with a tough leathery skin, longish snout with a curved horn sticking vertically up from the snout, small ears, piggish eyes, small tail, thick heavy legs with very distinct toes") reveals a significant contribution of information that is shared by other concepts. Moreover, it would in theory be possible to construct definitions of many concepts which meet the requirements of supplying a "core concept" using unique combinations of only shared attributes (e.g., "the large, strong African or Indian animal with tusks"). Previous studies (Devlin et al., 1998; McRae et al., 1997) have emphasized the importance of shared attributes, particularly in the representation of biological concepts. Shared visual attributes must also have a role in recognition, at least up to the point of assigning the item to the appropriate semantic category, and it is not surprising that these outnumber the distinctive attributes. The intuitive importance of distinctive information may therefore, in the present study, have been overshadowed by the simple numerical superiority of shared attributes.

In conclusion then, the study reported in this paper has introduced a method whereby semantic knowledge can be systematically quantified at a fine-grained level, and has shown that the performance on this probed knowledge task of patients at various stages of DAT is strikingly impaired. The idea that the anomia frequently observed in DAT reflects the disintegration of underlying semantic knowledge has been reinforced by the replication of the findings of Hodges et al. (1996) and by the additional demonstration of a graded relationship between naming success and amount of semantic knowl- 
edge available. Finally, a stronger relationship of increasing success at naming with visual than with functional knowledge has been demonstrated, arguing for the greater importance of visual knowledge to name production.

Although this last finding might be interpreted as providing some degree of support for the notion of a visually based semantic subsystem specialised for name production, it is also open to the rather more mundane explanation that naming from pictures (visual representations of concepts) relies more strongly on visual than on any other kind of knowledge. One response to this might be to examine the relationship between visual knowledge and naming in response to non-visual stimuli (such as verbal descriptions) and between visual knowledge and visually mediated tasks that do not involve name-retrieval (such as picture sorting and word-picture matching). In the absence of such data, a demonstration that the relationship between naming and visual knowledge differs between conceptual domains (such as living vs. non-living things), would constitute evidence that the role of visual information in naming was more "central" than "peripheral" - a view that was taken in a detailed study of changes in the content of concept definitions over time (Lambon Ralph, Patterson, \& Hodges, 1997). Although the number of cases available was too small for these analyses to be replicated here, further investigation of this issue, using the semantic attribute data and methods described in this paper, will form the subject of a future study.

\section{Acknowledgment}

This work was funded by means of Medical Research Council project grants to J.R.H. and fellowship awards to P.G.

\section{References}

Braak, H., \& Braak, E. (1991). Neuropathological staging of Alzheimer-related changes. Acta Neuropathologica, 82, 239-259.

Chertkow, H., \& Bub, D. (1990). Semantic memory loss in dementia of Alzheimer's type. Brain, 113, 397-417.

Devlin, J. T., Gonnerman, L. M., Andersen, E. S., \& Seidenberg, M. S. (1998). Category-specific semantic deficits in focal and widespread brain damage: A computational account. Journal of Cognitive Neuroscience, 10, 77-94.

Everitt, B. S., \& Dunn, G. (1991). Applied multivariate data analysis. London: Edward Arnold.

Farah, M. J., \& McClelland, J. L. (1994). A computational model of semantic memory impairment: Modality specificity and emergent category specificity. Journal of Experimental Psychology: General, 120, 339-357.

Folstein, M. F., Folstein, S. E., \& McHugh, P. R. (1975). Mini-mental state: A practical guide for grading the mental state of patients for the clinician. Journal of Psychiatric Research, 12, 189-198.

Garrard, P., Lambon Ralph, M. A., Watson, P. C., Powis, J., Patterson, K., \& Hodges, J. R. (2001a). Longitudinal profiles of semantic impairment for living and nonliving concepts in dementia of Alzheimer's type. Journal of Cognitive Neuroscience, 13, 892-909.

Garrard, P., Lambon Ralph, M. A., Hodges, J. R., \& Patterson, K. (2001b). Prototypicality, distinctiveness and intercorrelation: Analyses of the semantic attributes of living and nonliving concepts. Cognitive Neuropsychology, 18, 125-174.

Garrard, P., Lambon Ralph, M. A., \& Hodges, J. R. (2002). Semantic dementia: A category specific paradox. In E. M. E. Forde \& G. Humphreys (Eds.), Category-specificity in brain and mind (pp. 149-180). Hove: Psychology Press.

Gonnerman, L. M., Andersen, E. S., Devlin, J. T., Kempler, D., \& Seidenberg, M. S. (1997). Double dissociation of semantic categories in Alzheimer's disease. Brain and Language, 57, 254-279.

Greene, J. D. W., Hodges, J. R., \& Baddeley, A. D. (1995). Autobiographical memory and executive function in early dementia of Alzheimer type. Neuropsychologia, 33, 1647-1670.

Grober, E., Buschke, H., Kawas, C., \& Fuld, P. (1985). Impaired ranking of semantic attributes in dementia. Brain and Language, 26, 276-286.

Hodges, J. R., Patterson, K., Oxbury, S., \& Funnell, E. (1992a). Semantic dementia: Progressive fluent aphasia with temporal lobe atrophy. Brain, 115, 1783-1806.

Hodges, J. R., Salmon, D. P., \& Butters, N. (1992b). Semantic memory impairment in Alzheimer's disease: Failure of access or degraded knowledge?. Neuropsychologia, 30, 301-314.

Hodges, J. R., Graham, N., \& Patterson, K. (1995a). Charting the progression in semantic dementia: Implications for the organization of semantic memory. Memory, 3, 463-495.

Hodges, J. R., \& Patterson, K. (1995b). Is semantic memory consistently impaired early in the course of Alzheimer's disease. Neuroanatomical and diagnostic implications. Neuropsychologia, 33, 441-459.

Hodges, J. R., Patterson, K., Graham, N., \& Dawson, K. (1996). Naming and knowing in dementia of Alzheimer's type. Brain and Language, 54, 302-325.

Huff, F. J., Corkin, S., \& Growden, J. H. (1986). Semantic impairment and anomia in Alzheimer's disease. Brain and Language, 28, 235-249.

Laatu, S., Portin, R., Revonsuo, A., Tuisku, S., \& Rinne, J. (1997). Knowledge of concept meanings in Alzheimer's disease. Cortex, 33, $27-45$.

Lambon Ralph, M. A., Patterson, K. E., \& Hodges, J. R. (1997). The relationship between naming and semantic knowledge for different categories in dementia of Alzheimer's type. Neuropsychologia, 35, $1251-1260$.

Laws, K. R., Humber, S. A., Ramsey, D. J., \& McCarthy, R. A. (1995). Probing sensory and associative semantics for animals and objects in normal subjects. Memory, 3, 397-408.

Mathuranath, P. S., Nestor, P. J., Berrios, G. E., Rakowicz, W., \& Hodges, J. R. (2000). A brief cognitive test battery to differentiate Alzheimer's disease and frontotemporal dementia. Neurology, 55, 1613-1620.

McCarthy, R., \& Warrington, E. K. (1988). Evidence for modality specific meaning systems in the brain. Nature, 334, 428-430.

McKhan, G., Drachman, D., Folstein, M., Katzman, R., Price, D., \& Stadlan, E. M. (1984). Clinical diagnosis of Alzheimer's disease: Report of the NINCDS-ADRDA work group under the auspices of the Department of Health and Human Services Task Force on Alzheimer's disease. Neurology, 34, 939-944.

McRae, K., de Sa, V. R., \& Seidenberg, M. S. (1997). On the nature and scope of featural representations of word meaning. Journal of Experimental Psychology: General, 126, 99-130.

McRae, K., \& Cree, G. S. (2002). Factors underlying categoryspecific deficits. In E. M. E. Forde \& G. Humphreys (Eds.), Category-specificity in brain and mind (pp. 211-249). Hove: Psychology Press. 
Morrison, C. M., Chappell, T. D., \& Ellis, A. W. (1997). Age of acquisition norms for a large set of object names and their relation to adult estimates and other variables. Quarterly Journal of Experimental Psychology, 50, 528-559.

Moss, H. E., Tyler, L. K., Durrant-Peatfield, M., \& Bunn, E. M. (1998). Two eyes of a see-through: Impaired and intact semantic knowledge in a case of selective deficit for living things. Neurocase, 4, 339-352.

Nebes, R. D. (1989). Semantic memory in Alzheimer's disease. Psychological Bulletin, 106, 377-394.

Nebes, R. D., \& Brady, C. B. (1990). Preserved organisation of semantic attributes in Alzheimer's disease. Psychology and Aging, 5, 574-579.

Perry, R. J., \& Hodges, J. R. (1999). The spectrum of attentional dysfunction in dementia of Alzheimer's type. Brain, 122, 383-404.

Rogers, T. T., Lambon Ralph, M. A., Garrard, P., Bozeat, S., McClelland, J. L., Hodges, J. R., \& Patterson, K. (2004). The structure and deterioration of semantic memory: A neuropsycho- logical and computational investigation. Psychological Review, 111, 205-235.

Rosch, E. H. (1973). On the internal structure of perceptual and semantic categories. In T. E. Moore (Ed.), Cognitive development and the acquisition of language. New York: Academic Press.

Smith, E. E., \& Medin, D. L. (1981). Categories and concepts. Cambridge, MA: Harvard University Press.

Snodgrass, J. G., \& Vanderwart, M. A. (1980). A standardised set of 260 pictures: Norms for name agreement, familiarity and visual complexity. Journal of Experimental Psychology: General, 6, 174-215.

Warrington, E. K. (1975). The selective impairment of semantic memory. Quarterly Journal of Experimental Psychology, 27, 635-657.

Warrington, E. K. (1984). Recognition memory test. Windsor: NFER Nelson.

Warrington, E. K., \& Shallice, T. (1984). Category specific semantic impairments. Brain, 107, 829-853. 